\title{
COMMON FIXED POINT THEOREMS FOR UNIFORMLY SUBCOMPATIBLE MAPPINGS SATISFYING MORE GENERAL CONDITION
}

\author{
R. SUMITRA, V. RHYMEND UTHARIARAJ, P. VIJAYARAJU AND R. HEMAVATHY
}

\begin{abstract}
We prove common fixed point theorems for uniformly subcompatible mappings satisfying a more generalized Ćirić type condition and a condition more general than Gregus type condition in a locally convex domain. As an application, we have also established best approximation result. Our results extend recent results existing in the literature.
\end{abstract}

\section{Introduction}

Singh [16] proved fixed point and best approximation results in locally convex spaces. Hussain and Khan [9] employing a technique due to Tarafdar [17] obtained common fixed point results for a pair of noncommuting maps in the context of locally convex spaces.

Fisher and Sessa [5], Mukherjee and Verma [13] generalized the results of Gregus [6]. Jungck [7] introduced a new class of noncommuting mappings namely "Compatible mappings". Recently, Al-Mezel and Hussain [2] extended the concept of subcompatible mappings introduced by Khan et al. [10] to the setting of HausdorR locally convex space. Kutbi [12] and Akbar and Khan [1] proved common fixed point results for subcompatible pair of mappings satisfying generalized ciric type condition in the setup of locally convex space.

The purpose of this paper is to prove common fixed point theorems for uniformly subcompatible mappings satisfying a more general condition than generalized circ type, gregus type conditions in the setup of locally convex domain. This new concept extends various results existing in the literature.

Corresponding author: R. Sumitra.

Received September 18, 2009; revised May 5, 2010.

2000 Mathematics Subject Classification. 47H10 , 54H25.

Key words and phrases. Uniformly subcompatible mappings, generalized ciric type contractive condition, gregus type condition, common fixed point, best approximation, Locally convex space. 


\section{Definition and preliminaries}

Let $(X, \tau)$ be a Hausdorff locally convex topological vector space and $M$ be a $\tau$-bounded subset of $X$.

Definition 2.1. Let $(X, \tau)$ be a Hausdorff locally convex topological vector space. A family $\left\{p_{\alpha}: \alpha \in I\right\}$ of semi norms defined on $X$ is said to be an associated family of semi norms for $\tau$, if the family $\{\gamma U: \gamma>0\}$, where

$$
U=\bigcap_{i=1}^{n} U_{\alpha_{i}}, \quad U_{\alpha_{i}}=\left\{x: p_{\alpha_{i}}(x)<1\right\}
$$

forms a base of neighbourhoods of zero for $\tau$. Let the associated family of semi norms for $\tau$ be denoted as $A(\tau)$.

Definition 2.2. A family $\left\{p_{\alpha}: \alpha \in I\right\}$ of semi norms defined on $X$ is called an augmented associated family for $\tau$ if $\left\{p_{\alpha}: \alpha \in I\right\}$ is an associated family with the property that the semi norms $\max \left\{p_{\alpha}, p_{\beta}\right\} \in\left\{p_{\alpha}: \alpha \in I\right\}$ for any $\alpha, \beta \in I$. Let the augmented associated family of semi norms for $\tau$ be denoted by $A^{*}(\tau)$.

Definition 2.3. Asubset $M$ of $X$ is $\tau$-bounded iff each $p_{\alpha}$ is bounded on $M$.

Remark 2.1. ([9]) If $M$ is a $\tau$-bounded subset of $X$, then a number $\lambda_{\alpha}>0$, for each $\alpha \in I$ is selected such that $M \subset \lambda_{\alpha} U_{\alpha}$, where $U_{\alpha}=\left\{x: p_{\alpha}(x) \leq 1\right\}$. Clearly $B=\bigcap_{\alpha} \lambda_{a} U_{\alpha}$ is $\tau$-bounded, absolutely convex and contains $M$. The Minkowski functional of $B$ is a norm $\|\cdot\|_{B}$ on $X_{B}$. The space $\left(X_{B},\|\cdot\|_{B}\right)$ is a normed linear space with $B$ as its closed unit ball and

$$
\|x\|_{B}=\sup _{\alpha}\left\{p_{\alpha}\left(\frac{x}{\lambda_{\alpha}}\right)\right\}
$$

for each $x \in X_{B}$.

Definition 2.4. ([15]) Let $M$ be a nonempty $\tau$-bounded subset of $X$. The set of best $M$ approximants to $u \in X$, denoted as $P_{M}(u)$ is defined by

$$
P_{M}(u)=\left\{y \in M: p_{\alpha}(y-u)=\operatorname{dist}_{p_{\alpha}}(u, M) \text { for all } p_{\alpha} \in A^{*}(\tau)\right\},
$$

where dist $p_{\alpha}(u, M)=\inf \left\{p_{\alpha}(x-u): x \in M\right\}$.

Definition 2.5. ([3]) Let $f$ and $T$ be two self mappings of a nonempty $\tau$-bounded subset $M$ of a Hausdorff locally convex space $X$. The self mapping $T$ of $M$ is said to satisfy Ciric $f$ contractive type condition, if there exists real numbers $a, b, c$ with $0<a<1, b \geq 0, a+b=1$, $0 \leq c \leq \eta$ such that

$$
p_{\alpha}(T x-T y) \leq a \max \left\{p_{\alpha}(f x-f y), c\left[p_{\alpha}(f x-T y)+p_{\alpha}(f y-T x)\right]\right\}
$$




$$
+b \max \left\{p_{\alpha}(f x-T x), p_{\alpha}(f y-T y)\right\}
$$

for all $x, y \in M$ and for each $p_{\alpha} \in A^{*}(\tau)$, where $\eta=\min \left\{\frac{2+a}{5+a}, \frac{2-a}{4}, \frac{4}{9+a}\right\}$.

Definition 2.6. A self mapping $T$ of $M$ is said to be uniformly asymptotically regular on $M$ if for each $\varepsilon>0$, there exists $N(\varepsilon)=N$ such that $p_{\alpha}\left(T^{n} x-T^{n+1} x\right)<\varepsilon$ for all $n \geq N$, and $x \in M$.

Definition 2.7. A self mapping $T$ of a nonempty subset $M$ of a locally convex space $X$ is said to be

(1) compact, if $\left\{x_{n}\right\}$ is a bounded sequence in $M$, then $\left\{T x_{n}\right\}$ has a convergent subsequence $\left\{T x_{m}\right\}$ in $M$.

(2) hemicompact, if any sequence $\left\{x_{n}\right\}$ in $M$ has a convergent subsequence whenever $p_{\alpha}\left(x_{n}-\right.$ $\left.T x_{n}\right) \rightarrow 0$ as $n \rightarrow \infty$.

(3) demiclosed at 0 , if for every net $\left\{x_{\beta}\right\}$ in $M$ converging weakly to $x$ and $\left\{T x_{\beta}\right\}$ converging strongly to $0, T x=0$.

Definition 2.8. Two self mappings $T$ and $f$ of $X$ are said to be compatible, if for all $p_{\alpha} \in A^{*}(\tau)$, $\lim _{n} p_{\alpha}\left(T f x_{n}-f T x_{n}\right)=0$ whenever $\left\{x_{n}\right\}$ is a sequence such that $\lim _{n} T x_{n}=\lim _{n} f x_{n}=x_{0} \in$ $M$.

The following is the definition of subcompatible mappings recently given by Al-Mezel and Hussain [2] in the setup of locally convex space.

Definition 2.9. ([2]) Let $M$ be a $q$-starshaped subset of a Hausdorff topological locally convex space $X$. Let $f$ and $T$ be two self mappings of $M$ with $q \in F(f)$. The self mappings $f$ and $T$ of $M$ are said to be subcompatible, if

$$
\lim _{m} p_{\alpha}\left(f T x_{m}-T f x_{m}\right)=0
$$

for all sequences $\left\{x_{m}\right\} \in S_{q}(f, T)=\bigcup\left\{S\left(f, T_{k}\right): 0 \leq k \leq 1\right\}$ and for each $p_{\alpha} \in A^{*}(\tau)$, where $T_{k} x=$ $(1-k) q+k T x$ and $S\left(f, T_{k}\right)=\left\{\left\{x_{n}\right\} \in M: \lim _{n} f x_{n}=\lim _{n} T_{k} x_{n}=t \in M \Rightarrow \lim _{n} p_{\alpha}\left(f T_{k} x_{n}-\right.\right.$ $\left.\left.T_{k} f x_{n}\right)=0\right\}$.

It is well known that subcompatible pair of mappings is a compatible pair but not conversely in general which is seen from the following example in the setup of normed linear space. 
Example 2.1 . ([10]) Let $X=\mathbb{R}$ with usual norm and $M=[1, \infty)$. Let $f x=2 x-1$ and $T x=x^{2}$, for all $x \in M$. Then $M$ is $q$-starshaped with $q=1 \in F(f)$. It may be noted that $f$ and $T$ are compatible mappings as $\left\|f x_{n}-T x_{n}\right\|=0$ whenever $\left\{x_{n}\right\}$ is a sequence in $M$ such that $\lim _{n} f x_{n}=\lim _{n} T x_{n}=z$ for some $z \in M$. But $f$ and $T$ are not subcompatible mappings. Consider a sequence $\left\{x_{n}\right\}$ in $M$ with $\lim _{n} x_{n}=2$. Then $\lim f x_{n}=\lim _{n} T_{\frac{2}{3}} x_{n}=3 \in M$ and $\lim _{n}\left\|T x_{n} T f x_{n}\right\| \neq 0$.

\section{Main results}

On the same lines of Khan et al. [10], we define "uniformly subcompatible mappings" with slight modifications.

Definition 3.1. Let $f$ and $T$ be two self mappings of $q$-starshaped subset $M$ of a normed linear space $X$ with $q \in F(f)$. Define

$$
S_{q}\left(f, T^{n}\right)=\bigcup\left\{S\left(f, T_{k}\right): 0 \leq k \leq 1\right\},
$$

where $T_{k} x=(1-k) q+k T^{n} x$ and $S\left(f ; T_{k}\right)=\left\{\left\{x_{n}\right\} \subset M: \lim _{n} f x_{n}=\lim _{n} T_{k} x_{n}=z \in M \Rightarrow\right.$ $\left.\lim _{n}\left\|f T_{k} x_{n}-T_{k} f x_{n}\right\|=0\right\}$. The self mappings $f$ and $T$ of $M$ are said to be uniformly subcompatible, if $\lim _{m}\left\|f T^{n} x_{m}-T^{n} f x_{m}\right\|=0$ for all sequences $\left\{x_{m}\right\} \in S_{q}\left(f, T^{n}\right)$.

We give an example for "uniformly subcompatible mappings".

Example 3.2. Let $X=\mathbb{R}$ with usual norm and $M=[0, \infty)$. Let two self mappings $f$ and $T$ of $M$ be defined as

$$
\begin{aligned}
& f x=\left\{\begin{array}{ll}
\frac{\pi}{2} & \text { if } 0 \leq x<1 \\
2 x^{2}-1 & \text { if } x \geq 1
\end{array}\right. \text { and } \\
& T x=\left\{\begin{array}{ll}
\frac{1}{2} & \text { if } 0 \leq x<1 \\
4 x-3 & \text { if } x \geq 1
\end{array} .\right.
\end{aligned}
$$

Then $M$ is $q$-starshaped with $q=1 \in F(f)$ and $S_{q}\left(f, T^{n}\right)=\bigcup\left\{S\left(f, T_{k}\right): 0 \leq k \leq 1\right\}=\left\{\left\{x_{n}\right\}: 1 \leq\right.$ $x_{n}<\infty$ \}. It may be noted that $f$ and $T$ are uniformly subcompatible mappings. But $f$ and $T$ are not uniformly $R$-subweakly commuting.

Now we give the definition of "uniformly subcompatible mappings" in the setup of locally convex space. 
Definition 3.2. Let $M$ be a $q$-starshaped subset of a Hausdorff locally convex space $(X, \tau)$. Let $f$ and $T$ be two self mappings of $M$ with $q \in F(f)$. Define $\left.S_{q}\left(f, T^{n}\right)=\bigcup\left(f, T_{k}\right): 0 \leq k \leq 1\right\}$, where $T_{k} x=(1-k) q+k T^{n} x$ and $S\left(f, T_{k}\right)=\left\{\left\{x_{n} g\right\} \subset M: \lim _{n} f x_{n}=\lim _{n} T_{k} x_{n}=z \in M \Rightarrow\right.$ $\left.\lim _{n} p_{\alpha}\left(f T_{k} x_{n}-T_{k} f x_{n}\right)=0\right\}$. The self mappings $f$ and $T$ of $M$ are said to be uniformly subcompatible, if

$$
\lim _{m} p_{\alpha}\left(f T^{n} x_{m}-T^{n} f x_{m}\right)=0
$$

for all sequences $\left\{x_{m}\right\} \in S_{q}\left(f, T^{n}\right)$ and for each $p_{\alpha} \in A^{*}(\tau)$.

The following lemma of Kutbi [12] will be needed in the sequel.

Lemma 3.1. ([12]) Let $M$ be a nonempty $\tau$-bounded, $\tau$-sequentially complete and convex subset of a Hausdorff locally convex space $(X, \tau)$. Let $f, T: M \rightarrow M$ be compatible self mappings of $M$ and $T(M) \subseteq f(M)$. If $T$ is continuous and Ciric $f$-contractive type mapping satisfying inequality (??), then $f$ and $T$ have a unique common solution.

Theorem 3.1. Let $T$ and $f$ be self mappings of a nonempty $\tau$-bounded, $\tau$-complete and $q$ starshaped subset $M$ of a Hausdorfflocally convex space $(X, \tau)$. Suppose that $f$ is $A^{*}(\tau)$-nonexpansive and affine w.r.t $q \in F(f)$ and $c l[T(M)] \subseteq f(M)$. If $T$ and $f$ are uniformly subcompatible on $M$ satisfying,

$$
\begin{aligned}
p_{\alpha}\left(T^{n} x-T^{n} y\right) \leq & \max \left\{p_{\alpha}(f x-f y), c\left[\operatorname{dist}_{p_{\alpha}}\left(f x,\left[T^{n} y, q\right]\right)+\operatorname{dist}_{p_{\alpha}}\left(f y,\left[T^{n} x, q\right]\right)\right]\right\} \\
& +\frac{1-k}{k} \max \left\{\operatorname{dist}_{p_{\alpha}}\left(f x,\left[T^{n} x, q\right]\right), \operatorname{dist}_{p_{\alpha}}\left(f y,\left[T^{n} y, q\right]\right)\right\},
\end{aligned}
$$

for all $x, y \in M$, for each $p_{\alpha} \in A^{*}(\tau), n=1,2,3, \ldots, \infty$ and $0 \leq c \leq \eta$ and $k \in(0,1)$, where $\eta=$ $\min \left\{\frac{2+k}{5+k}, \frac{2-k}{4}, \frac{4}{9+k}\right\}$, then $T$ and $f$ have a common fixed point provided one of the following conditions hold:

(i) $\operatorname{cl}[T(M)]$ is $\tau$-sequentially compact.

(ii) $M$ is weakly compact and $f-T^{n}$ is demiclosed at 0 .

Proof. Define a mapping $T_{n}$ as $T_{n} x=\left(1-k_{n}\right) q+k_{n} T^{n} x$ for all $x \in M$ and for each $n \geq 1$, where $\left\{k_{n}\right\}$ is a sequence of real numbers such that $0<k_{n}<1$ and $k_{n} \rightarrow 1$ as $n \rightarrow \infty$.

As $M$ is $q$-starshaped, $f$ is affine with respect to $q \in F(f)$ and $c l[T(M)] \subseteq f(M)$, it follows that each $T_{n}$ is a well defined self mapping of $M$ and $\operatorname{cl}\left[T_{n}(M)\right] \subseteq f(M)$ for each $n$. Moreover, $T_{n} f x=k_{n} T^{n} f x+\left(1-k_{n}\right) q$ and $f T_{n} x=k_{n} f T^{n} x+\left(1-k_{n}\right) q$.

Since $f$ and $T$ are uniformly subcompatible on $M$, there exists a sequence $\left\{x_{m}\right\} \in S_{q}\left(f, T_{n}\right)$ such that $\lim _{m} f x_{m}=\lim _{m} T_{n} x_{m}=z \in M$ and which satisfy

$$
\lim _{m} p_{\alpha}\left(T_{n} f x_{m}-f T_{n} x_{m}\right)=k_{n} \lim _{m} p_{\alpha}\left(T^{n} f x_{m}-f T^{n} x_{m}\right)=0 .
$$


Thus the pair $\left\{T_{n}, f\right\}$ is compatible for each $n=1,2,3, \ldots, \infty$. Further, as $T$ satisfies inequality (3.1), it follows that

$$
\begin{aligned}
& p_{\alpha}\left(T_{n} x-T_{n} y\right)=k_{n} p_{\alpha}\left(T^{n} x-T^{n} y\right) \\
& \leq k_{n}\left[\max \left\{p_{\alpha}(f x-f y), c\left[\operatorname{dist}_{p_{\alpha}}\left(f x,\left[T^{n} y, q\right]\right)+\operatorname{dist}_{p_{\alpha}}\left(f y,\left[T^{n} x, q\right]\right)\right]\right\}\right. \\
&\left.+\frac{1-k_{n}}{k_{n}} \max \left\{\operatorname{dist}_{p_{\alpha}}\left(f x,\left[T^{n} x, q\right]\right), \operatorname{dist}_{p_{\alpha}}\left(f y,\left[T^{n} y, q\right]\right)\right\}\right] \\
& \leq k_{n} \max \left\{p_{\alpha}(f x-f y), c\left[\operatorname{dist}_{p_{\alpha}}\left(f x,\left[T^{n} y, q\right]\right)+\operatorname{dist}_{p_{\alpha}}\left(f y,\left[T^{n} x, q\right]\right)\right]\right\} \\
&+\left(1-k_{n}\right) \max \left\{\operatorname{dist}_{p_{\alpha}}\left(f x,\left[T^{n} x, q\right]\right), \operatorname{dist}_{p_{\alpha}}\left(f y,\left[T^{n} y, q\right]\right)\right\}
\end{aligned}
$$

for all $x, y \in M$, for each $p_{\alpha} \in A^{*}(\tau)$ and $0<k_{n}<1$.

(i) As $\operatorname{cl}[T(M)]$ is $\tau$-sequentially compact, each $c l\left[T_{n}(M)\right]$ is also $\tau$-sequentially compact and hence is $\tau$-sequentially complete. Now the pair $\left(T_{n}, f\right)$ satisfies all the conditions of Lemma 3.1. Hence there exists $x_{n} \in M$ such that $f x_{n}=x_{n}=T_{n} x_{n}=\left(1-k_{n}\right) q+k_{n} T^{n} x_{n}$. Thus

$$
x_{n}-T^{n} x_{n}=\left(1-k_{n}\right)\left(q-T^{n} x_{n}\right) .
$$

Since $M$ is $\tau$-bounded, $\left\{T^{n} x_{n}\right\}$ is a bounded sequence. As $k_{n} \rightarrow 1$ as $n \rightarrow \infty$, it follows that $p_{\alpha}\left(x_{n}-T^{n} x_{n}\right) \rightarrow 0$ as $n \rightarrow \infty$.

As $T$ is uniformly asymptotically regular, there exists $N(\varepsilon)=N$ such that $p_{\alpha}\left(T^{n} x_{n}-\right.$ $\left.T^{n+1} x_{n}\right)<\varepsilon$ for all $n \geq N$. Hence $p_{\alpha}\left(T^{n} x_{n}-T^{n+1} x_{n}\right) \rightarrow 0$ as $n \rightarrow \infty$, which implies that

$$
p_{\alpha}\left(x_{n}-T^{n+1} x_{n}\right) \leq p_{\alpha}\left(x_{n}-T^{n} x_{n}\right)+p_{\alpha}\left(T^{n} x_{n}-T^{n+1} x_{n}\right) \rightarrow 0 \text { as } n \rightarrow \infty .
$$

As $\left\{T^{n} x_{n}\right\}$ is a sequence in $\operatorname{cl}[T(M-\{q\})]$, which is $\tau$-sequentially compact, there exists a subsequence $\left\{T^{m} x_{m}\right\}$ of $\left\{T^{n} x_{n}\right\}$ such that $T^{m} x_{m} \rightarrow x_{0}$ as $m \rightarrow \infty$ for some $x_{0} \in M$. Moreover, since $k_{m} \rightarrow 1$ as $m \rightarrow \infty$, it follows that

$$
x_{m}=f x_{m}=\left(1-k_{m}\right) q+k_{m} T^{m} x_{m} \rightarrow x_{0} \text { as } m \rightarrow \infty .
$$

Since $f$ is continuous, $x_{m}=f x_{m} \rightarrow f x_{0}$ as $m \rightarrow \infty$. By the uniqueness of the limit $x_{0}=$ $f x_{0}$.

As $T$ is continuous, $T^{m} x_{m} \rightarrow T^{m} x_{0}$ as $m \rightarrow \infty$. Again by the uniqueness of the limit

$$
\begin{aligned}
\lim _{m \rightarrow \infty} T^{m} x_{0} & =x_{0} \\
\lim _{m \rightarrow \infty} T^{m+1} x_{0} & =T x_{0} .
\end{aligned}
$$

Now, by equations (3.4) and (3.5) and also by the uniformly asymptotically regularity of $T$, it follows that

$$
p_{\alpha}\left(x_{0}-T x_{0}\right) \leq p_{\alpha}\left(x_{0}-T^{m} x_{0}\right)+p_{\alpha}\left(T^{m} x_{0}-T^{m+1} x_{0}\right)+p_{\alpha}\left(T^{m+1} x_{0}-T x_{0}\right) \rightarrow 0 \text { as } n \rightarrow \infty .
$$

Therefore $x_{0}=T x_{0}$. Thus $x_{0}=f x_{0}=T x_{0}$. 
(ii) As in (i), there exists $x_{n} \in M$ such that $T_{n} x_{n}=f x_{n}=x_{n}$. Since $M$ is weakly compact, there exists a subsequence $\left\{x_{m}\right\}$ of $\left\{x_{n}\right\}$ converging weakly to some $x_{0} \in M$. As $f$ being affine with respect to $q$ and continuous is weakly continuous and since the weak topology is Hausdorff, it follows that $f x_{0}=x_{0}$. Moreover, by inequality (3.2) we have

$$
\lim _{m} p_{\alpha}\left(f x_{m}-T^{m} x_{m}\right)=\lim _{m} p_{\alpha}\left(x_{m}-T^{m} x_{m}\right)=0 .
$$

Now the demiclosedness of $\left(f-T^{m}\right)$ at 0 guarantees that $\left(f-T^{m}\right) x_{0}=0$. Hence $x_{0}=$ $T^{m} x_{0}=f x_{0}$. Proceeding as in (i), we have $x_{0}=f x_{0}=T x_{0}$.

The following corollary substantially improves Theorems of [2], [5], [6] and [13] to the setup of locally convex space and more general condition than Gregus type condition.

Corollary 3.1. Let $T$ and $f$ be self mappings of a nonempty $\tau$-bounded, $\tau$-complete and $q$ starshaped subset $M$ of a Hausdorfflocally convex space $(X, \tau)$. Suppose that $f$ is $A^{*}(\tau)$-nonexpansive and affine w.r.t $q \in F(f)$ and $c l[T(M)] \subseteq f(M)$. If $T$ and $f$ are uniformly subcompatible on $M$ satisfying,

$$
p_{\alpha}\left(T^{n} x-T^{n} y\right) \leq p_{\alpha}(f x-f y)+\frac{1-k}{k} \max \left\{\operatorname{dist}_{p_{\alpha}}\left(f x,\left[T^{n} x, q\right]\right), \operatorname{dist}_{p_{\alpha}}\left(f y,\left[T^{n} y, q\right]\right)\right\},
$$

for all $x ; y \in M$, for each $p_{\alpha} \in A^{*}(\tau), n=1,2,3, \ldots, \infty$, then $T$ and $f$ have a common äed point provided one of the conditions (i) -(ii) of Theorem 3.1 hold.

Proof. The proof of the Corollary 3.1 follows by taking $c=0$ in Theorem 3.1.

Theorem 3.2. Let $T, f$ and $g$ be self mappings of a nonempty $\tau$-bounded, $\tau$-complete and $q$ starshaped subset $M$ of a Hausdorff locally convex space $(X, \tau)$. Suppose that $T$ is continuous, $q \in F(f) \cap F(g), f$ and $g$ are $A^{*}(\tau)$-nonexpansive and affine w.r.tq. If $c l[T(M)] \subseteq f(M) \cap g(M)$, the pairs $\{T, f\}$ and $\{T, g\}$ are uniformly subcompatible,

$$
p_{\alpha}\left(T^{n} x-T^{n} y\right) \leq p_{\alpha}(f x-g y)+\frac{1-k}{k} \max \left\{\operatorname{dist}_{p_{\alpha}}\left(f x,\left[T^{n} x, q\right]\right), \operatorname{dist}_{p_{\alpha}}\left(g y,\left[T^{n} y, q\right]\right)\right\}
$$

for all $x, y \in M$, for each $p_{\alpha} \in A^{*}(\tau)$ and $n=1,2,3, \ldots, \infty$, then $F(T) \cap F(f) \cap F(g) \cap M$ is singleton provided one of the following conditions hold:

(i) $\operatorname{cl}[T(M)]$ is $\tau$-sequentially compact.

(ii) $M$ is weakly compact and $f-T^{n}$ is demiclosed at 0 .

Remark 3.1. Theorem 3.1 and Theorem 3.2 extends very recent result of Al-Mezel and Hussain [2] to the setting of more generalized gregus type condition and extends very recent result of Akbar and Khan [1] to the setting of generalized asymptotically nonexpansive mappings. They also generalize main theorems of [2], [9], [12], [14], [17]. 


\section{Applications to best approximations}

Theorem 4.1. Let $T$ and $f$ be two self continuous mappings of a Hausdorff locally convex space $(X, \tau)$ with $u \in F(T) \cap F(f)$. Let $M$ be a nonempty subset of $X$ such that $T(\partial M \cap M) \subseteq M$, where $\partial M$ is the boundary of $M$. Suppose that $P_{M}(u)$ is $\tau$-bounded, closed and q-starshaped, $f$ is $A^{*}(\tau)$-nonexpansive and affine with respect to $q \in F(f)$ with $f\left(P_{M}(u)\right)=P_{M}(u)$ and $T$ is uniformly asymptotically regular and satisfy inequality (3.1) on $P_{M}(u)$. If $T$ and $f$ are uniformly subcompatible on $P_{M}(u) \cup\{u\}$ satisfying $p_{\alpha}(T x-T u) \leq p_{\alpha}(f x-f u)$, then $T$ and $f$ have a unique common fixed point in $P_{M}(u)$ provided

(i) $\operatorname{cl}\left[T\left(P_{M}(u)\right)\right]$ is $\tau$-sequentially compact.

(ii) $P_{M}(u)$ is weakly compact, $\tau$-sequentially complete and $f-T^{n}$ is demiclosed at 0 .

Proof. Let $x \in P_{M}(u)$. Then $p_{\alpha}(x-u)=d_{p_{\alpha}}(u, M)=\inf \left\{p_{\alpha}(y-u): y \in M\right\}$ for each $p_{\alpha} \in A^{*}(\tau)$.

For any $k \in(0,1), p_{\alpha}(k u+(1-k) x-u)=(1-k) p_{\alpha}(x-u)<d_{p_{\alpha}}(u, M)$. It follows the line segment $\{k u+(1-k) x: 0 \leq k \leq 1\}$ and the set $M$ are disjoint. Thus $x$ is not in the interior of $M$ and so $x \in \partial M \cap M$. Since $T(\partial M \cap M) \subseteq M$, it follows $T x \in M$. Moreover $f\left(P_{M}(u)\right)=P_{M}(u)$ implies $f x \in P_{M}(u)$. As $u \in F(T) \cap F(f)$, we have

$$
p_{\alpha}(T x-u) \leq p_{\alpha}(T x-T u) \leq p_{\alpha}(f x-f u)=p_{\alpha}(f x-u)=d_{p_{\alpha}}(u, M)
$$

which implies $T x \in P_{M}(u)$. Hence $T\left(P_{M}(u)\right) \subseteq P_{M}(u)=f\left(P_{M}(u)\right)$. By applying Theorem 3.1, there exists $z \in P_{M}(u)$ such that $z=f z=T z$.

Remark 4.1. Theorem 4.1 generalizes main theorems of [2], [9], [12], [14], [17].

Corollary 4.1. Assuming all the conditions of Theorem 4.1 to be same except the mapping $T$ satisfy inequality (3.6) instead of inequality (3.1), then also $T$ and $f$ have a unique common fixed point in $P_{M}(u)$.

Proof. Proof. The proof of the Corollary 4.1 follows by taking $c=0$ in Theorem 4.1.

\section{References}

[1] F. Akbar and A. R. Khan, Common fixed point and approximation results for noncommuting maps on locally convex spaces, Fixed point theory and applications, 2009(2009), article ID 207503, pp.14 pages.

[2] S. Al-Mezel and N. Hussain, On common fixed point and approximation results of gregus type, Int. Math. Forum, 2 (2007), 1839-1847.

[3] G. V. R. Babu and K. N. V. V. Prasad, Common fixed point theorems of diRerent compatible type mappings Ciric's contraction type condition, Mathematical Communications, 11(2006), 87-102.

[4] Lj. B. Ciric, On a common fixed point theorem of a Gregus type, Publ. Inst. Math., 49(1991), 174-178. 
[5] B. Fisher and S. Sessa, On a fixed point theorem of Gregus, Int. J. Math. Math. Sci., 9(1986), 23-28.

[6] M. Gregus, Jr., A fixed point theorem in Banach space, Bull. Un Mat. Ital., 5(1980), 193-198.

[7] G. Jungck, Compatible mappings and common fixed points, Internat. J. Math. Math. Sci., 9(1986), 43-49.

[8] G. Jungck, Common fixed points for commuting and compatible maps on compacta, Proceedings of the American Mathematical Soceity, 103(1988), 977-983.

[9] N. Hussain and A. R. Khan, Common fixed point results in best approximation theory, Applied Mathematics Letters, 16(2003), 575-580.

[10] A. R. Khan, F. Akbar and N. Sultana, Random coincidence points of subcompatible multivalued maps with applications, Carpathian Journal of Mathematics, 24(2008), 63-71.

[11] G. Kothe, Topological Vector Spaces, Springer verlag, New York, 1969.

[12] M. A. Kutbi, On common solution of contractive-type maps, Int. J. Contemporary Math. Sci., 4(2009), 541-548.

[13] R. N. Mukherjee and V. Verma, A note on fixed point theorem of gregus, Math. Japon., 33(1988), 745-749.

[14] H. K. Nashine and M. S. Khan, An application of fixed point theorem to best approximation in locally convex space, Appl. Math. Letters, in press 2009.

[15] S. Reich, Approximate selections, best approximations, fixed point and invariant sets, J. Math. Anal. Appl., 62(1978), 104-113.

[16] S. P. Singh, Some results on best approximation in locally convex spaces, J. Approx. Theory., 28(1980), 329-332.

[17] E. Tarafdar, Some fixed point theorems on locally convex linar topological spaces, Bull. Austral. Math. Soc., 13 (1975), 241-254.

[18] P. Vijayaraju, Fixed point theorems for asymptotically nonexpansive mapping, Bull. Calcutta Math. Soc., 80(1998), 133-136.

Department of Mathematics, SMK Fomra Institute of Technology, Chennai-603 103, Tamilnadu, India.

E-mail: suhemaths@rediRmail.com

Ramanujan Computing Centre, Anna University, Chennai-600 025, Tamilnadu, India.

E-mail: rhymend@annauniv.edu

Department of Mathematics, Anna University, Chennai-600 025, Tamilnadu, India.

E-mail: vijay@annauniv.edu

Department of Mathematics, Easwari Engineering College, Ramapuram, Chennai-600 089, Tamilnadu, India.

E-mail: hemaths@rediRmail.com 